ACCEPTED MANUSCRIPT

\title{
Design Criteria and Applications of Multi-channel Parallel Microfluidic Module
}

To cite this article before publication: Yichao Huang et al $2018 \mathrm{~J}$. Micromech. Microeng. in press https://doi.org/10.1088/1361-6439/aad746

\section{Manuscript version: Accepted Manuscript}

Accepted Manuscript is "the version of the article accepted for publication including all changes made as a result of the peer review process, and which may also include the addition to the article by IOP Publishing of a header, an article ID, a cover sheet and/or an 'Accepted

Manuscript' watermark, but excluding any other editing, typesetting or other changes made by IOP Publishing and/or its licensors"

This Accepted Manuscript is @ 2018 IOP Publishing Ltd.

During the embargo period (the 12 month period from the publication of the Version of Record of this article), the Accepted Manuscript is fully protected by copyright and cannot be reused or reposted elsewhere.

As the Version of Record of this article is going to be / has been published on a subscription basis, this Accepted Manuscript is available for reuse under a CC BY-NC-ND 3.0 licence after the 12 month embargo period.

After the embargo period, everyone is permitted to use copy and redistribute this article for non-commercial purposes only, provided that they adhere to all the terms of the licence https://creativecommons.org/licences/by-nc-nd/3.0

Although reasonable endeavours have been taken to obtain all necessary permissions from third parties to include their copyrighted content within this article, their full citation and copyright line may not be present in this Accepted Manuscript version. Before using any content from this article, please refer to the Version of Record on IOPscience once published for full citation and copyright details, as permissions will likely be required. All third party content is fully copyright protected, unless specifically stated otherwise in the figure caption in the Version of Record.

View the article online for updates and enhancements. 


\title{
Design Criteria and Applications of Multi-channel Parallel Microfluidic Module
}

\author{
Yichao Huang ${ }^{1}$, Tengteng Han ${ }^{1}$, Jin Xuan ${ }^{2}$, Hong $\mathrm{Xu}^{1}$, Yongle Wang ${ }^{1}$, Li Zhang ${ }^{1 *}$ \\ ${ }^{1}$ State-Key Laboratory of Chemical Engineering, School of Mechanical and Power Engineering, East \\ China University of Science and Technology, Shanghai, 200237, China \\ ${ }^{2}$ Department of Chemical Engineering, Loughborough University, Loughborough, LE11 3TU, United \\ Kingdom
}

*Corresponding Author E-mail: lzhang@ecust.edu.cn (L. Zhang).

\begin{abstract}
The microfluidic technology for function microspheres synthesis has high control precision. However, the throughput is too low for industrial scale-up applications. Current scale-up design focuses on mutli-chanel in two dimensions, in which the distribution uniformity parameter $\delta$ increases linearly, resulting in the deterioration of the flow distribution performance. The three-dimensional modular scale-up strategy could greatly alleviate this problem, but no design principles have been developed yet. In the first time, this paper established the microfluidic three-dimensional scale-up design criteria. Based on the modular design concept, the design method of two and three-dimensional throughput scale-up parameters $N$ and $M$, distribution uniformity parameters $\delta$ and $\beta$, and microchannel design parameter $K_{R}$ were proposed. The equivalent resistance coefficient was defined, and the influence of different parameters in two-dimensional array and three-dimensional stack was analyzed. Furthermore, the error correction method was studied. It was found that the twostage scale-up process contradicted each other. A good scale-up performance of one stage led to the limitation of another stage. Increasing the resistance of each channel $R_{u}$ could both increase two-stage scale-up performance, which was an important factor. A single-module scale-up system with 8 channels in a single array and 10 arrays in a vertical stack, which had 80 channels in total, was designed and fabricated based on the proposed design criteria for generating Chitosan $/ \mathrm{TiO}_{2}$ composite microspheres. The average particle size was $539.65 \mu \mathrm{m}$ and $\mathrm{CV}$ value was about $3.59 \%$. The throughput was $480 \mathrm{ml} / \mathrm{h}$, which effectively increased the throughput scale and the product quality.
\end{abstract}

Keywords: Microfluidic, Scale-up design criteria, Modular design, Flow resistance module.

\section{Intruduction}

Microfluidics is undergoing rapid development in recent years which can be applied in various fields such as chemistry, biology, medicine, etc [1]. It is acknowledged as controlling fluids accurately on the microscale [2]. Compared with the traditional techniques, microfluidic technology can be used to synthesis microdroplets with uniform size and controllable structure [3-7]. Microdroplets of different structures and materials have a wide range of applications in many fields [811].

Despite the obvious benefits, most researches are confined to the laboratory scale, which focus on exploring the new microfluidic chips with different structure to have a better control of size, component and material of microparticles. However, they ignore the low throughput and other practical obstacles which are related to the applications in industrial production. Fortunately, many researchers and companies 
have carried out relevant studies in an attempt to solve the difficulties brought about by the large-scale industrialization, such as the benefits, microchannel structure and production rate [12].

For the challenge of low production rate, the general method is to use multiple channels in parallel arrangement [13]. The appropriate structure can keep the flow in each branch as uniform as possible [14], which is the basis for the scale-up strategy. Many large-scale droplet generation devices of different structures have been published [15-20]. The twodimensional and three-dimensional scale-up are two scale-up forms.

Two-dimensional scale-up is currently the main form, and its structure is simple. Therefore, most researchers chose to parallel a large number of channels in two dimensions for large-scale droplet generation $[16,19,20]$. When designing a scale-up structure, it is necessary to satisfy the flow distribution uniformity and fully consider the parameters matching for various design. Some researchers have proposed corresponding scale-up design criteria based on the twodimensional ladder-like network of microchannels and applied it to the design of microfluidic double emulsion devices. They connected 15 channels in parallel, and the droplet throughput reach $1 \mathrm{~kg} /$ day. The coefficient of variation is less than 6\% [21]. Later, some researchers also applied it on a $3 \mathrm{~cm} \times 3 \mathrm{~cm}$ PDMS microfluidic chip to integrate 512 drop makers with the particle size of about $86.1 \mu \mathrm{m}$ [22] and integrated up to 1000 flow focusing generators with production rate of $1.51 / \mathrm{h}$ [23].

However, a large number of microchannels in parallel in two dimensions will also bring some hidden trouble. The twodimensional and three-dimensional scale-up effects have been compared. The two-dimensional scale-up uniformity parameter $\delta$ is proportional to the number of channels $N$. The increased $N$ leads to the increased $\delta$ which reflects the deterioration of the flow distribution performance. With the same number of channels, the three-dimensional scale-up stacking process makes much less increasement of uniformity parameter $\delta$ or $\beta$ than it in two dimensions, which can effectively limit the deterioration of distribution performance [24].

Therefore, three-dimensional scale-up has adyantages over two dimensions for the same throughput. At present, there are some three-dimensional structures for high-throughput generation. A stackable microfluidic droplet generator consisting of 512 droplet makers on 4 layers with a particle size of about $200 \mu \mathrm{m}$ has been published [15]. There has also been a modular scale-up, in which 16 parallel droplet makers are arranged in a single module. And it used the integration of multiple modules to amplify with the production rate of $50 \mathrm{~g} / \mathrm{h}$ [17].

On the other hand, the parameters that need to be considered in the three-dimensional structure are more complicated than the two-dimensional ones. The matching of different scale-up parameters leads to the various product quality. Some researchers have realized this problem. They studied the effects of different scale-up parameter proportioning on the final distribution performance from two-dimensional to multidimensional scale-up and the effects of different dimension on distribution performance under the same throughput [25]. In spite of this, no three-dimensional scale-up design criterion have been proposed, from which the specific suggestions for parameter selection can be made based on actual requirements and conditions to obtain optimal results.

In response to the above problems, we proposed a set of new design criteria for three-dimensional scale-up in this paper. Combining ideas from two-dimensional arrays, threedimensional stacks to the modular integration [24], the design principle was presented. At the same time, various parameters involved in three-dimensional scale-up were studied, and the influence of various parameters was analyzed to provide guidance for the optimization of scale-up performance. Based on the proposed design criteria, a microfluidic droplet generation module with 80 channels was designed and optimized. The monodispersity of the prepared Chitosan $/ \mathrm{TiO}_{2}$ composite microspheres was excellent, which verified the effectiveness and reliability of the proposed design criteria.

\section{Methods}

\subsection{Flow resistance model}

The flow resistance model in a single module was used for analysis, including the flow resistance among neighboring channel inlets $\left(R_{f}\right)$, the resistance of each channel $\left(R_{u}\right)$, the equivalent resistance of a single array $\left(R_{c}\right)$, the flow resistance of vertical channels among neighboring arrays $\left(R_{v}\right)$, and the motivation in a fluid gravitational field $\left(R_{g}\right)$, as shown in Fig. 1. In order to achieve uniform flow distribution in the threedimensional microfluidic system, $R_{u}$ should be significantly greater than $R_{f}$ while $R_{c}$ should be also much greater than $\mid R_{v^{-}}$ $R_{g} \mid$. Based on the above rules, it is possible to derive the influencing parameters and the modular scale-up design criteria.

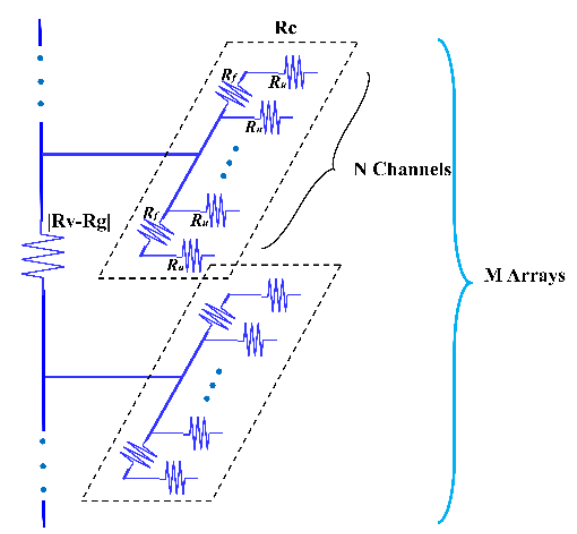

Fig. 1. Model for the hydraulic resistance of a three-dimensional microfluidic network

\subsection{Equivalent resistance of a single array}


The $R_{c}$ is an important parameter which is a bridge that connects two-dimensional parallel array and threedimensional modular scale-up. Mathematical and physical methods can be used to calculate $R_{c}$.

The design criteria of the two-dimensional arrays in ladder geometry have been proposed and demonstrated for the uniform flow distribution [21-23]:

\section{$2 N\left(R_{f} / R_{u}\right) \leq 0.01$}

In order to reveal the intrinsic relationship among scale-up influencing parameters, flow uniformity and microchannel structure, the above design criterion was also modified based on the ladder-like distribution. The two parameters $\delta$ and $\beta$ were used to characterize the flow distribution performance. The smaller values of these two parameters are, the more excellent the flow distribution performance is, and the more uniform the particle size is. The Eq. 1 can be modified as Eq. 2 and Eq. 3 for the two-dimensional array and threedimensional stack. $N$ is the number of channels in the array and $M$ is the number of arrays in the stack.

$N\left(\frac{R_{f}}{R_{l}}\right)=\delta$

$M\left(\frac{\left|R_{v}-R_{g}\right|}{R_{c}}\right)=\beta$

The coefficient k was used instead of $\delta / N$, and the $R_{f}$ can be derived from Eq. 4:

$R_{f}=\frac{\delta R_{u}}{N}=k R_{u}$

The mathematical formula was used to describe the twodimensional resistance model in Fig. 1, as shown in Eq. 5, where $R_{c, N}$ and $R_{c, N-1}$ was the equivalent resistances of $N$ channels and $N-1$ channels.

$R_{c, N}=k R_{u}+\frac{R_{u} R_{c, N-1}}{R_{u}+R_{C, N-1}}$

Solve the above general formula, and finally get mathematic relationship for $R_{c, N}$, as shown in Eq. 6:

$R_{c, N}=\left[\frac{k}{2}+\left(\frac{f^{N}(k)+1}{f^{N}(k)-1}\right) \frac{\sqrt{k^{2}+4 k}}{2}\right] R_{u}$

where $f(k)=\frac{k+2+\sqrt{k^{2}+4 k}}{k+2-\sqrt{k^{2}+4 k}}$.

$\xi=\left[\frac{k}{2}+\left(\frac{f^{N}(k)+1}{f^{N}(k)-1}\right) \frac{\sqrt{k^{2}+4 k}}{2}\right]=f\left(\frac{\delta}{N}, N\right)$ is the equivalent resistance coefficient of the array, then Eq. 6 can be simplified as shown in Eq. 7:

$\left.R_{c, N}=\xi R_{u}=f\left(\frac{\delta}{N}, N\right) R_{u}\right)$

The calculation of the flow resistance $R$, is analogous to the basic principle of the circuit, since the flow of the fluid in the channel is similar to the transfer of the current in the wire.
According to the ohmic formula $R=U / I$, the single channel resistance can be get as shown in Eq. 8, where $R_{\text {channel }}$ is the flow resistance of the channel, $d P$ is the pressure drop between the inlet and outlet, and $Q$ is the flow rate of the channel.

$R_{\text {channel }}=\frac{d P}{Q}$

$R_{i}$ is the resistance of a circular channel under laminar flow which is determined by channel diameter, length and fluid viscosity. We can use Eq. 9 to estimate it:

$R_{i}=\frac{128 \mu l}{\pi D_{h}^{4}}=\frac{8 \mu l}{\pi r^{4}}$

\section{Results and discussion}

\subsection{Relationship between equivalent resistance efficient and two-dimensional array}

It is found that $R_{c, N}$ is related to $\xi$ and $R_{u}$ from the definition of equivalent resistance in the two-dimensional array. Either of these two parameters increase will lead to the increment of $R_{c, N}$. The equivalent resistance coefficient $\xi$, which is an integrated function of $N$ and $\delta / N$, is related to two-dimensional parallelization number $N$ and the parameter of flow distribution performance $\delta . \delta$ is mainly affected by the structure of the distribution, such as the parallel array or the circular array. Since $\xi$ is a complex physical parameter, it needs to be discussed in detail. The relationship among $\xi, N$ and $\delta$ in the two-dimensional array is studied.

$N$ directly affects the production rate. The higher $N$ indicates the higher production rate. $\delta$ directly affects the uniformity of distribution. The smaller $\delta$ results in the higher uniformity. Therefore, in the two-dimensional array, it is necessary to obtain a higher value of $N$ and a smaller value of $\delta$. To further study their internal relationship, we have got Fig.2(created by Matlab) which is the curve family of $\xi$ changing with $\delta / N$ and $N$. In the figure, the $\mathrm{X}$-axis is $\delta / N$ while the $\mathrm{Y}$-axis is $\xi$. For example, if we parallelize 8 channels in two-dimensional array, $N$ will be 8 and a purple curve will be selected. Different $\delta$ are selected to obtain different $\xi$. As can be seen from the figure, when $N$ is small, the correlation among $\xi, \delta$ and $N$ is obvious. Higher $\delta$ indicates the higher $\xi$. But increasing $N$ will lead to a smaller $\xi$. With the increase of $N$, the curve tends to be gentle, and the influence of $\delta$ on $\xi$ becomes lower, mainly depending on the value of $N$. When $N$ equals 64 or more, these curves basically coincide and achieve convergent. Then $\xi$ converges to 0.0733 , and it has the lowest correlation with $\delta$ and $N$.

Therefore, in the two-dimensional array, if the value of $N$ is high enough, the $\xi$ is the minimum and the curve is gentle, regardless of $N$ and the $\delta$; if the value of $N$ is limited, $N$ and $\delta$ should be considered to determine $\xi$, and then calculate the value of equivalent resistance. 


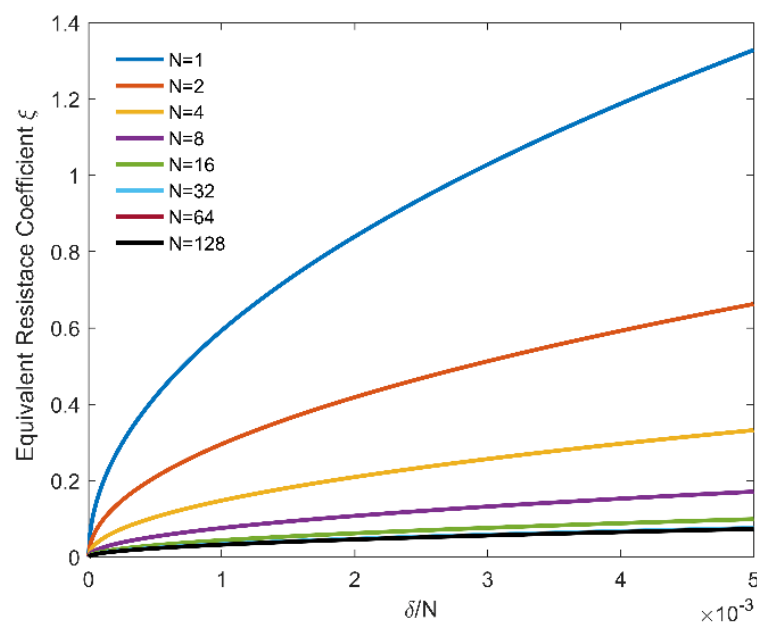

Fig. 2. The equivalent resistance coefficient $\xi$ with different $N$ and $\delta / N$

\subsection{Relationship between equivalent resistance efficient and three-dimensional stack}

Substitute Eq. 3 of design criterion in three-dimensional stack into the Eq. 7 of equivalent resistance. Eq. 10 can be obtained:

$\frac{\left|R_{v}-R_{g}\right| / R_{u}}{\beta / M}=\xi$

Similar to $N$ and $\delta$ of two-dimensional array, $M$ is the number of vertically stacked arrays. It directly affects the droplet throughput. The higher $M$ will lead to the higher throughput. $\beta$ is an indicator of vertical flow distribution performance. The smaller $\beta$ value results in higher uniform distribution. Therefore, in the three-dimensional stack, it is also necessary to obtain a higher value of $M$ and a smaller value of $\beta$.

Eq. 10 can be modified as Eq. 11 , where $K_{R}=\left|R_{v}-R_{g}\right| /$ $R_{u}$ is defined as the design parameter of the stack/module. $K_{R}$ means the ratio of the resistance of vertical flow distribution channel to the resistance of single channel, which is the core in designing the single channels and the vertical flow distribution channels. The smaller $K_{R}$ leads to the more conservative channel design. On the other word, it is much easier to obtain the higher throughput and better uniformity.

$\frac{K_{R}}{\beta / M}=\xi$

Eq. 11 reveals the relationship between $\xi$ and the threedimensional stack. Fig. 3 is the curve family of $\xi$ changing with $\beta / M$ and $K_{R}$ (created by Matlab). It can be seen from the figure that, for the determined $K_{R}$, the smaller the $\xi$ leads to the higher $\beta / M$. The smaller $K_{R}$, which results in the more conservative design, leads to the smaller $\beta / M$ with the same $\xi$. Therefore, to obtain the same value of $\beta$, the number of stacked array $M$ can be increased appropriately. When the value of $K_{R}$ is conservative enough to be below $5 \times 10^{-5}$, several curves are basically coincided. Therefore, it is not necessary to get further conservative design. A higher level of processing difficulty is related to the more conservative design. When $K_{R}$ and $\xi$ is constant, for a fixed $\beta / M$ value, the higher uniformity means that we should decrease $\beta$ as well as $M$, or we can decrease the uniformity $(\beta$ increases $)$ to get a higher $M$.

Equivalent resistance is an important parameter that relates two-dimensional array and three-dimensional stack, and $\xi$ can also reflect the performance of two-stage scale-up. From the perspective of $\xi$, in the two-dimensional array, it is desirable to increase $N$ and lower $\delta$ to achieve higher output and better uniformity, but the result is to achieve a small $\xi$. When the $\xi$ is small, the fixed $K_{R}$ in the three-dimensional stack will result in a higher $\beta / M$. It is actually detrimental because we need to get a smaller $\beta / M$ to accommodate the higher throughput with the higher $M$ and the higher uniformity with the smaller $\beta$. It can be seen that the requirements of two-stage scale-up for $\xi$ are in conflict. According to Eq. 2 and Eq. 3, it can also be seen that increasing $R_{u}$ can get smaller $\delta$ and $\beta$ at the same time, which is good for two-stage scale-up. However, the improvement of $R_{u}$ means the increased difficulty of mechanical processing and the channel structure design. In practice, combined with the mechanical processing and design conditions, we candetermine $R_{u}$ as much as possible. And then according to the parameter $N, \xi$ can be determined from Fig. 2 . $\beta / M$ can be determined by certain $K_{R}$ and $\xi$ in Fig. 3. The values of $\beta$ and $M$ should be properly assigned.

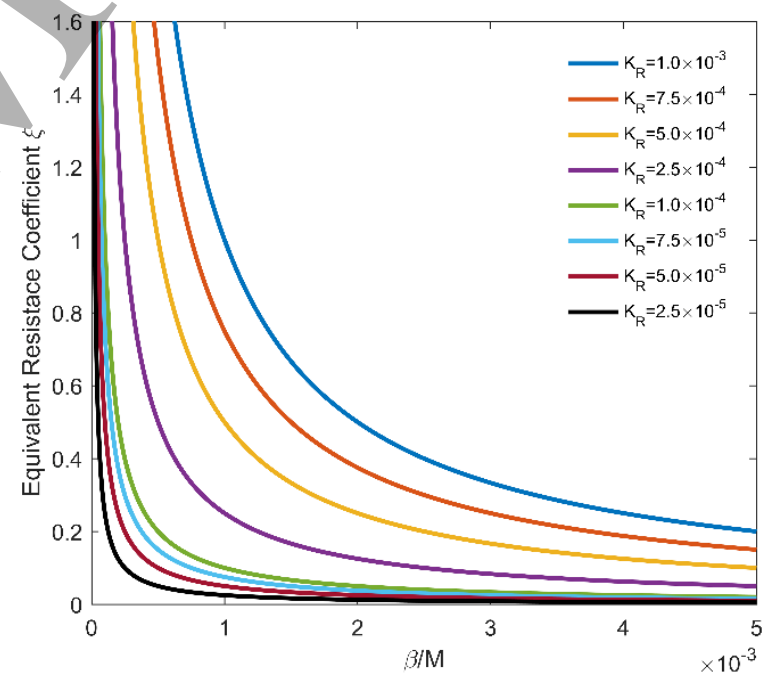

Fig. 3. The equivalent resistance coefficient $\xi$ with different $K_{R}$ and $\beta / M$

\subsection{Multi-stage module design criteria}

The multi-stage module design criterion Eq. 12 can be got by combining Eq. 7 with Eq. 11:

$\frac{K_{R}}{\beta / M}=f\left(\frac{\delta}{N}, N\right)$ 
In this criterion, there are three categories of parameters, which are the throughput scale-up parameters $M$ and $N$, the distribution uniformity parameters $\delta$ and $\beta$, and the microchannel design parameter $K_{R}$. The two throughput scaleup parameters are directly related to the whole throughput. Higher $M$ and $N$ lead to higher throughput. The two distribution uniformity parameters characterize the distribution performance of three-dimensional multi-stage scale-up module. The smaller two values are, the more uniform the distribution performance is. And the microchannel design parameter is related to the machining geometric parameters. The smaller value indicates the more conservative design, but it will be subject to processing accuracy, space volume and other factors. We discussed these parameters separately based on the derived multi-stage module design criterion. Since $\delta$ and $\beta$ have the same meaning, for convenience, we made these two values equal.

The relationship between throughput scale-up parameters and microchannel design parameters was discussed. Better uniformity can be achieved while setting the two uniformity parameters $\delta$ and $\beta$ to 0.005 . Fig. 4 shows the curve family of $M$ changing with different $N$ and $K_{R}$ while $\delta$ and $\beta$ are fixed at 0.005 (created by Matlab). For different $K_{R}\left(K_{R}\right.$ is respectively $2.5 \times 10^{-5}, 5 \times 10^{-5}, 7.5 \times 10^{-5}, 1 \times 10^{-4}, 2.5 \times 10^{-4}, 5 \times 10^{-4}, 7.5 \times 10^{-4}$, $1 \times 10^{-3}$.), the selected value of $M, N$ is different. The higher $K_{R}$ indicates that the curve is closer to the coordinate axes, and the values of $M$ and $N$ will be selected more strictly. Conversely, smaller $K_{R}$ indicates the more conservative design, so throughput scale-up parameters may be appropriate to increase. For either case, the maximum of $M$ can be determined from the Fig. 4 after selecting parameter $N$. Or according to the demand for throughput scale-up parameters, $K_{R}$ can be determined by the graph, and then it is used to guide the microchannel design. It can also be found from the figure that when increasing $R_{u}$, considering that $\delta$ and $\beta$ are fixed values, $N$ and $M$ will be further improved as $K_{R}$ decreases.

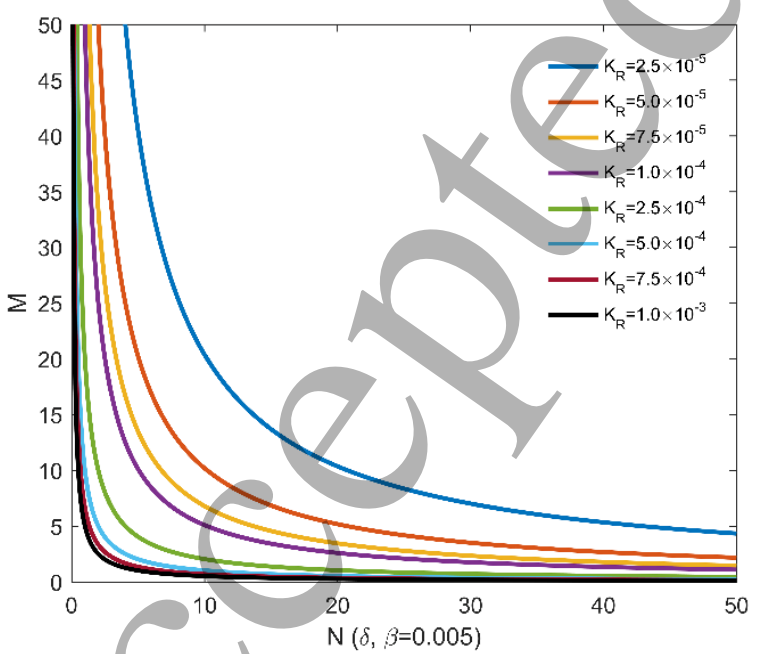

Fig. 4. Three-dimensional throughput scale-up parameters $M$ with different $N$ and $K_{R}(\delta$ and $\beta$ are fixed at 0.005$)$
On the other hand, the relationship between throughput scale-up parameters and uniformity parameters was discussed. As shown in Fig.5(created by Matlab), $K_{R}$ is set to be $2.5 \times 10^{-}$ ${ }^{4}$. For different uniformity requirements, the values of $M$ and $N$ are also different. Fig.5 is the curve family of $M$ changing with different uniformity parameters while $K_{R}$ is fixed at $2.5 \times 10^{-4}$ (Both $\delta$ and $\beta$ values are $0.02,0.015,0.01,0.008$, $0.006,0.004,0.002$, and 0.001). As can be seen from the figure, when $K_{R}$ is fixed, the higher uniformity requirements leads to a lower value of $\delta$ and $\beta$. Meanwhile, the value of $M$ and $N$ are selected more strictly and the increment of throughput is more limited. The appropriate increment of $\delta$ and $\beta$ (flow distribution performance degradation) can greatly enhance the throughput. Based on the value of $M$ and $N$, the distribution uniformity can be roughly determined.

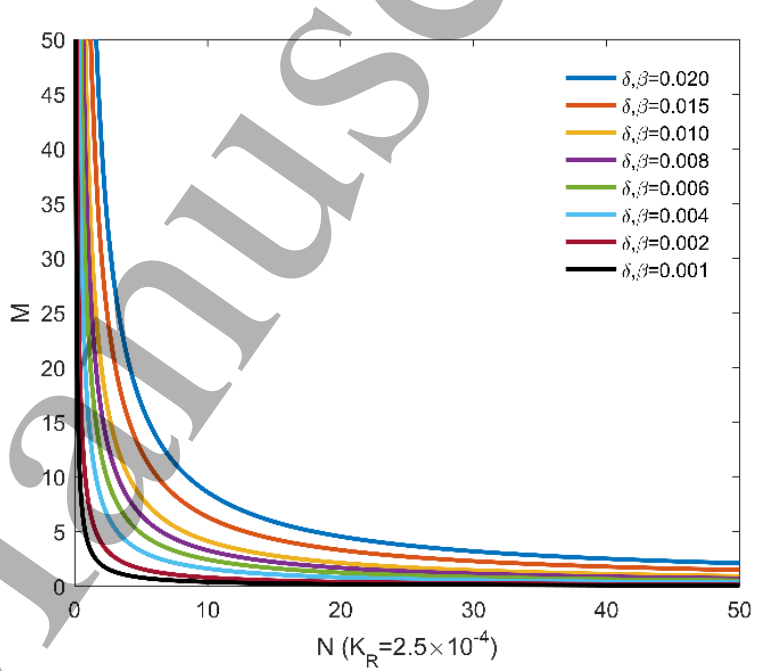

Fig. 5. Three-dimensional throughput scale-up parameters $M$ with different $N, \delta$ and $\beta$ ( $K_{R}$ is fixed at $2.5 \times 10^{-4}$ )

Fig. 6 is production throughput $(N \times M)$ changing with different $\delta, \beta$ and $K_{R}$. The $\mathrm{Y}$-axis is the product of twodimensional parallelization number $N$ and the threedimensional stack number $M$, which is $N \times M . N$ is fixed at 8 . The value of $N \times M$ is directly related to the production throughput. The dispersed phase flow rate of single unit $Q_{c s}$ multiplies by the total number of units $N \times M$, and $Q_{c s} \times N \times M$ is the production throughput of a single module. For convenience, we set two distribution uniformity parameters $\delta=\beta$ since they have the same meaning. It can be quickly seen from Fig. 6 that the increment of throughput $(N \times M)$ directly leads to an increment of the values of $\delta$ and $\beta$, which refers to the deterioration of the flow distribution performance. To improve the flow distribution performance, the values of $\delta$ and $\beta$ need to be reduced, and then $N \times M$ decreases. Therefore, it can be found that the demand for scale-up and the uniformity of flow distribution are in conflict. In addition, while $\delta$ and $\beta$ are kept constant, smaller $K_{R}$ will lead to the obvious increment of $N \times M$. The result is consistent with the discussion in section 3.2. Smaller $K_{R}$ reflects the improvement of $R_{u}$ 
(resistance of each channel), which is related to the mechanical processing and the channel structure design.

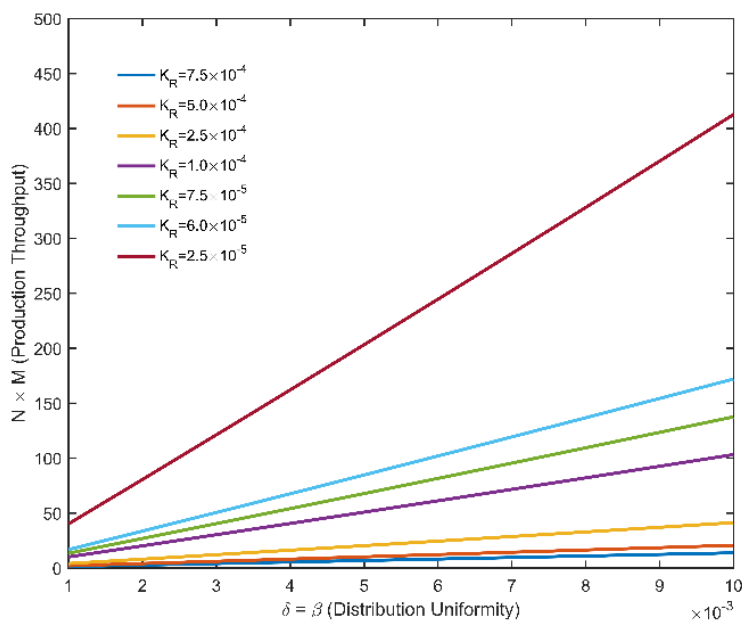

Fig.6 Production throughput $(N \times M)$ with different $\delta, \beta$ and $K_{R}(N$ is fixed at 8 and $\delta=\beta$ )

\subsection{Correction of criteria}

The above analysis was based on the ideal condition, and the flow resistance model based on ladder-shaped asymmetric structure was deduced without considering the influence of the machining error. Therefore, it is necessary to modify the above derivation process. Two basic criteria Eq. 2 and Eq. 3 are modified to Eq. 13 and Eq. 14 respectively:

$N\left[\frac{\left(R_{f}+\Delta R_{u}\right)}{R_{u}}\right]=\delta$

$M\left[\frac{\left(\left|R_{v}-R_{g}\right|+\Delta R_{u}\right)}{R_{C}}\right]=\beta$

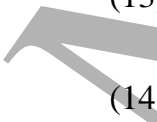

Where $\Delta R_{u}$ is the error of microchannel resistance $R_{u}$ in machining process, which is the main influencing factor of flow distribution. Through resolving Eq. 13, Eq. 15 can be obtained.

$\frac{R_{f}}{R_{u}}+\frac{\Delta R_{u}}{R_{u}}=\frac{\delta}{N}$

The left side of Eq. 15 is composed of two terms, $\frac{R_{f}}{R_{u}}$ and $\frac{\Delta R_{u}}{R_{u}}$, respectively, revealing two key issues of fluid distribution. $\frac{R_{f}}{R_{u}}$ is the structural issue, which refers to the asymmetry factor of the branch structure. And it mainly reflects the pressure difference among neighboring channel inlets. $\frac{\Delta R_{u}}{R_{u}}$ is nonstructural issue, which refers to machining process error and it is not related to the structure. To meet the uniformity of flow distribution, some conditions should be achieved:

Structural condition: The resistance among neighboring channel inlets $R_{f}$ should be far less than the resistance of each channel $R_{u}$ so as to ensure that the inlet pressure of each channel is nearly the same.

Non-structural conditions: The error of the resistance of each branch is negligible compared with $R_{u}$ so as to ensure that each $R_{u}$ is nearly the same.

Eq. 14 can also be split in a similar way, as shown in Eq. 16:

$\frac{\left|R_{v}-R_{g}\right|}{\xi R_{u}}+\frac{\Delta R_{u}}{\xi R_{u}}=\frac{\beta}{M}$

When achieving the completely symmetrical ladder-like structure, the distribution channel evolves into a central reservoir. And the pressure of each branch is almost the same, which means reducing $R_{f}$ in the maximum extent and reaching the limit of structural condition. Therefore, the distribution uniformity mainly depends on the processing error of the microchannel. In order to meet the non-structural conditions, the serpentine microchannel needs to be adopted to improve the resistance of each branch as shown in Fig.7, so that the flow uniform distribution can be conveniently achieved. As the length of the serpentine channel increases, the distribution performance will also gradually increase. Our research team has presented a flow distributor based on polymethylmethacrylate (PMMA) and laser microfabrication engraving (Universal VLS2.30) [24], which uses serpentine channel [26]. Serpentine channel can balance the pressure drop at each branch inlet and the non-uniform flow resistance caused by machining process to improve flow distribution performance [27-29].
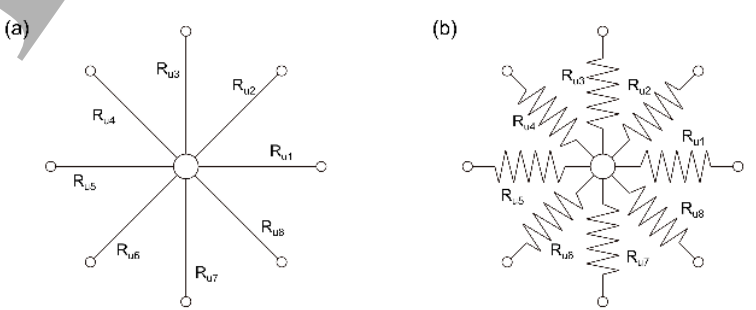

Fig. 7. The completely symmetrical structure: (a) straight channel; (b) serpentine channel

\subsection{Experimental verification}

According to the design criteria proposed in this paper, the design and manufacture of scale-up module have been carried out. In the discussion of the previous section, a long serpentine channel was adopted to meet the non-structural condition, and a circular array was adopted to meet the structural condition.

The experiment, taking into account the processing equipment and structure factor, selected $80 \mathrm{~mm} \times 80 \mathrm{~mm}$ polymethylmethacrylate (PMMA) plate to array 8 channels. Therefore $N=8$ was selected. It has been studied that in twodimensional scale-up criterion, $2 \delta$ should be less than 0.01 so that flow distribution performance is better, hence $\delta=0.005$ is 
selected [21-23]. As discussed in Section 3.1 and shown in Fig. $2, \xi \approx 0.13$ was obtained.

The above parameters belong to two-dimensional scale-up parameters, and then three-dimensional scale-up parameters were discussed. Due to the limitation of PMMA plate size and processing precision, the total length of the serpentine channel was set to be approximately $280 \mathrm{~mm}$, and the channel section was a small triangle with the size of $619 \mu \mathrm{m}$ which was viewed by an optical microscope with a digital camera (OLYMPUS SZX7). Based on the actual processing size, the $R_{u}$ value can be calculated, and then $K_{R}$ can be got. Through the above parameters calculated, it shows that $\beta / M$ is a certain value by Eq. 11 and Fig.3. From Fig.1, the three-dimensional scale-up can also be reduced to the two-dimensional scale-up. Therefore, we set $\beta<0.005$. When $\beta$ is less than or equal to 0.005 , the performance of flow distribution in the threedimensional scale-up is better. Fig. 8 is the value of $\beta$ changing with $M$. When $M=10$ is set, $\beta$ is just less than 0.005 while $\beta$ is 0.005197 with $M=11$, meeting the flow distribution uniformity requirements. According to the above steps, we determined $N$ $=8, M=10$.

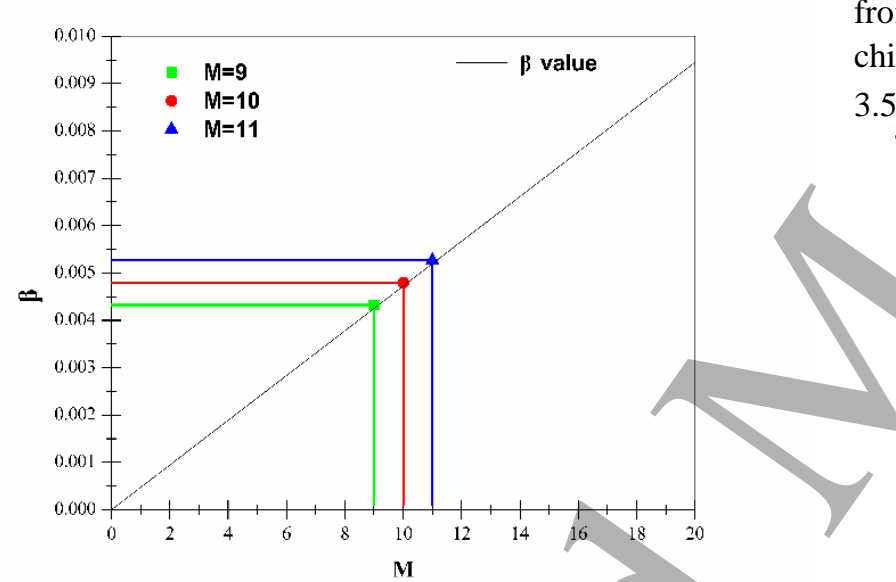

Fig. 8. $\beta$ changing with $M(M=9, \beta=0.004252 ; M=10, \beta=0.004725$; $M=11, \beta=0.005197)$

The relevant verification experiments have been carried out by our research team. According to the design criteria, a scaleup module has been manufactured. Taking chitosan system as an example, the chitosan/ $\mathrm{TiO}_{2}$ composite microspheres were prepared and the particle size, surface morphology, CV Values were also analysed. The detailed experiment has been reported by our research team [24]. The chitosan powder and $\mathrm{TiO}_{2}$ powder were dissolved in acetic acid (Shanghai Lingfeng Chemical Reagents Co., Ltd.) and deionized water, forming an aqueous solution as the dispersed phase. The continuous phase was made from a mixture of paraffin liquid and petroleum ether with volume ratio of 7:5 (all from Shanghai Aladdin Bio-Chem Technology Co., LTD), and 5\% span 80 was added as stabilization for chitosan droplets. The functional microspheres prepared by chitosan have good adsorption properties and they can be widely used in industrial wastewater treatment, ion adsorption and other fields [30-34].
The experiment schematic and the photograph of experimental equipment are shown in Fig.9(a) and Fig.9(b). The preparation process of chitosan system is shown in Fig.9(c). The specific structure can refer to the patent [35]. Before the new test started, the necessary parameters could be adjusted, such as solution composition, experimental temperature and so on. The experimental parameters should be kept constant during the test. Between run to run, the variations that could be controlled were the flow rate of the dispersed phase and continuous phase. The matching of the two-phase flow determines the size of droplets. The scale-up module was run at a continuous phase flow rate $Q_{c}$ of 80 $\mathrm{mL} / \mathrm{min}$ and a dispersed phase flow rate $Q_{d}$ of $8 \mathrm{~mL} / \mathrm{min}$ with two-phase flow rate ratio $Q_{d} / Q_{d}$ of 10 . The production throughput of a single module was approximately $12 \mathrm{~L} /$ day. $7.3 \times 10^{5}$ droplets could be generated per hour in a single module. Fig.10 shows the particle size of the microspheres prepared by the module. The number on the $\mathrm{X}$-axis means the two-dimensional scale-up array from top to bottom. Due to the vertical stacking of the arrays, $Q_{c}$ increases while $Q_{d}$ decreases with the particle diameter of each array generally decreasing from the first array to the tenth array. The average diameter of chitosan microspheres is $539.65 \mu \mathrm{m}$ and the $\mathrm{CV}$ value is about $3.59 \%$.

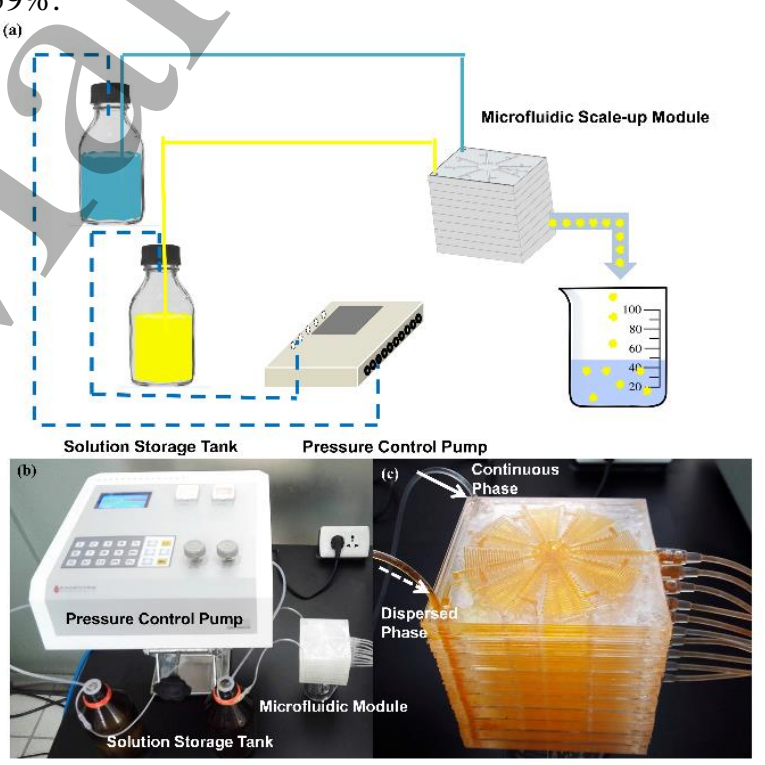

Fig. 9. Scale-up module experiment: (a) Experiment schematic; (b) A photograph of experimental equipment; (c) The preparation process of chitosan system. 


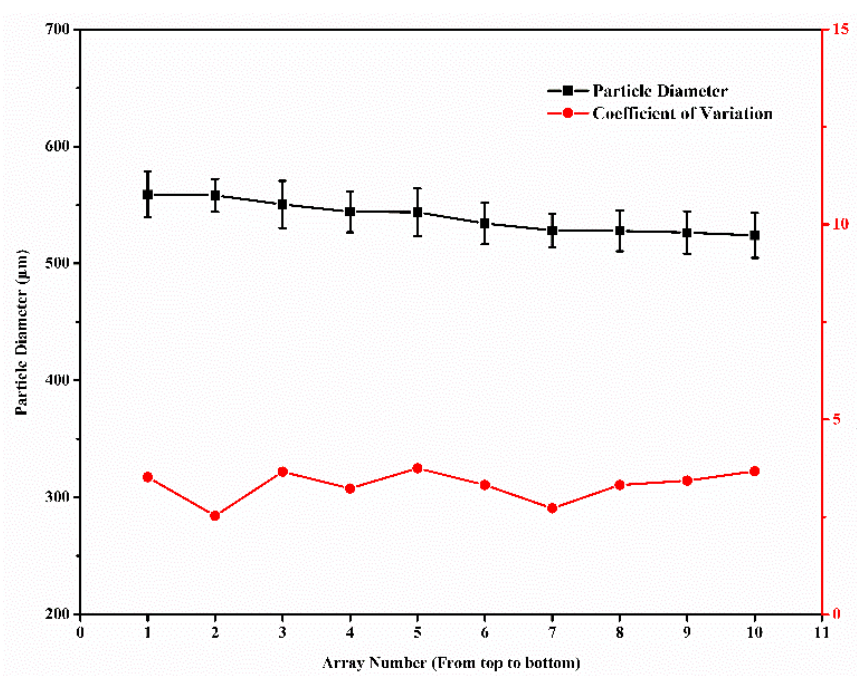

Fig. 10. The particle size of the microspheres prepared by the module (The first array refers to the top one)

Obviously, many modules can be integrated to build a microfluidic scale-up system. The flow distribution error is related to the processing error of each module and the flow rate error of fluid driving devices.

\section{Conclusion}

In this paper, based on the microfluidic modular design concept of two-dimensional array and three-dimensional stack, we have proposed three-dimensional scale-up design criteria.

A flow resistance theoretical model for the pressure balance of the complicated flow network inside the microfluidic module is established. The equivalent resistance coefficient is defined and its relationship with two-dimensional array parameters and three-dimensional stack parameters has been analyzed. In addition, the microchannel design parameter $K_{R}$ is presented. According to the relationship among the channel design parameters, the throughput scale-up parameters and the distribution uniformity parameters, the design of scale-up module can be guided. It is found that the resistance of each channel $R_{u}$ is a key factor affecting the uniformity of flow distribution in the process of module scale-up. Increasing $R_{u}$ indicates the improvement of both two-stage scale-up effect at the same time.

The microfluidic module has been designed and manufactured by applying the design criteria, and the largescale synthesis of monodisperse Chitosan/Titania composite microspheres is achieved. The reliability of the proposed three-dimensional microfluidic modular design criteria has been verified and the important theoretical guidance has been provided for the design of microfluidic devices which generate high-throughput functional microsphere materials.

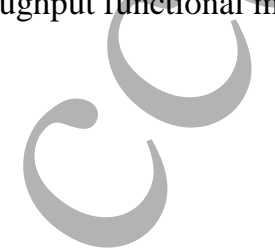

\section{Acknowledgements}

The research work is supported by the research grant (No. 16DZ2260600) from Science and Technology Commission of Shanghai Municipality, and the Fundamental Research Funds for the Central Universities" (222201717012) 222201718005)

\section{References}

[1] Tu S T, Yu X, Luan W and Löwe H 2010 Development of micro chemical, biological and thermal systems in China: A review Chemical Engineering Journal 163 165-79

[2] Ahmed I, Iqbal H M N and Akram Z 2017 Microfluidics Engineering: Recent Trends, Valorization, and Applications Arabian Journal for Science \& Engineering x 1-10

[3] Anna S L 2016 Droplets and Bubbles in Microfluidic Devices Annual Review of Fluid Mechanics 48 págs. 285-309

[4] Keohane K, Brennan D, Galvin P and Griffin B T 2014 Silicon microfluidic flow focusing devices for the production of sizecontrolled PLGA based drug loaded microparticles International Journal of Pharmaceutics 46760

[5] Li E Q, Zhang J M and Thoroddsen S T 2013 Simple and inexpensive microfluidic devices for the generation of monodisperse multiple emulsions Journal of Micromechanics \& Microengineering 24 563-90

[6] Tirandazi P and Hidrovo C H 2017 Liquid-in-gas droplet microfluidics; experimental characterization of droplet morphology, generation frequency, and monodispersity in a flow-focusing microfluidic device Journal of Micromechanics \& Microengineering 27

[7] Yeh C H and Lin Y C 2013 Use of an adjustable microfluidic droplet generator to produce uniform emulsions with different concentrations Journal of Micromechanics \& Microengineering 235025

[8] Gong X, Peng S, Wen W, Sheng P and Li W 2009 Design and Fabrication of Magnetically Functionalized Core/Shell Microspheres for Smart Drug Delivery Advanced Functional Materials 19 292-7

[9] Li X, Iwai K, Pirmoradi F N, Yang C and Lin L 2015 Controlled drug delivery via remotely heated core-shell magnetic microcapsules. In: Transducers - 2015 International Conference on Solid-State Sensors, Actuators and Microsystems, pp 1049-52

[10] Park J H, Han C M, Lee E J and Kim H W 2016 Preparation of highly monodispersed porous-channeled poly(caprolactone) microspheres by a microfluidic system Materials Letters 181 92-8

[11] Wang W, Zhang M J and Chu L Y 2014 Functional Polymeric Microparticles Engineered from Controllable Microfluidic Emulsions Accounts of Chemical Research 47373

[12] Holtze C 2013 Large-scale droplet production in microfluidic devices - an industrial perspective Journal of Physics D Applied Physics 46114008

[13] Ehrfeld W, Hessel V and Löwe H 2001 Microreactors: New Technology for Modern Chemistry

[14] Nisisako T 2016 Recent advances in microfluidic production of Janus droplets and particles Current Opinion in Colloid \& Interface Science 25 1-12 
[15] Conchouso D, Castro D, Khan S A and Foulds I G 2014 Threedimensional parallelization of microfluidic droplet generators for a litre per hour volume production of single emulsions Lab on A Chip 14 3011-20

[16] Femmer T, Jans A, Eswein R, Anwar N, Moeller M, Wessling $\mathrm{M}$ and Kuehne A J 2015 High-Throughput Generation of Emulsions and Microgels in Parallelized Microfluidic DropMakers Prepared by Rapid Prototyping Acs Appl Mater Interfaces 7 12635-8

[17] Li W, Greener J, Voicu D and Kumacheva E 2009 Multiple modular microfluidic (M3) reactors for the synthesis of polymer particles Lab on A Chip 9 2715-21

[18] Nisisako T and Torii T 2008 Microfluidic large-scale integration on a chip for mass production of monodisperse droplets and particles Lab on A Chip 8 287-93

[19] Ofner A, Moore D G, Rühs P A, Schwendimann P, Eggersdorfer M, Amstad E, Weitz D A and Studart A R 2017 High-Throughput Step Emulsification for the Production of Functional Materials Using a Glass Microfluidic Device Macromolecular Chemistry \& Physics 2181600472

[20] Vladisavljević G T, Ekanem E E, Zhang Z, Khalid N, Kobayashi I and Nakajima M 2018 Long-term stability of droplet production by microchannel (step) emulsification in microfluidic silicon chips with large number of terraced microchannels Chemical Engineering Journal 333 380-91

[21] Romanowsky M B, Abate A R, Rotem A, Holtze C and Weitz D A 2012 High throughput production of single core double emulsions in a parallelized microfluidic device Lab on A Chip 12 802-7

[22] Muluneh M and Issadore D 2013 Hybrid soft-lithography/laser machined microchips for the parallel generation of droplets Lab on A Chip 134750

[23] Jeong H H, Yelleswarapu V R, Yadavali S, Issadore D and Lee D 2015 Kilo-scale droplet generation in three-dimensional monolithic elastomer device (3D MED) Lab on A Chip 154387

[24] Han T, Zhang L, Xu H and Xuan J 2017 Factory-on-chip: Modularised microfluidic reactors for continuous mass production of functional materials \$ Chemical Engineering Journal 326

[25] Commenge J M, Saber M and Falk L 2011 Methodology for multi-scale design of isothermal laminar flow networks Chemical Engineering Journal 173 541-51

[26] Wang H, Gu S, Leung D Y C, Xu H, Leung M K H, Zhang L and Xuan J 2014 Development and characteristics of a membraneless microfluidic fuel cell array Electrochimica Acta 135 467-77

[27] Jeon N L, Dertinger S K W, Chiu D T, Choi I S, And A D S and Whitesides G M 2000 Generation of Solution and Surface Gradients Using Microfluidic Systems Langmuir 16 8311-6

[28] Kim C, Lee K, Kim J H, Shin K S, Lee K J, Kim T S and Kang J Y 2008 A serial dilution microfluidic device using a ladder network generating logarithmic or linear concentrations Lab on A Chip 8 473-9

[29] Oh K W, Lee K, Ahn B and Furlani E P 2012 Design of pressure-driven microfluidic networks using electric circuit analogy Lab on A Chip 12515

[30] Dong Z, Xu H, Bai Z, Wang H, Zhang L, Luo X, Tang Z, Luque R and Xuan J 2015 Microfluidic synthesis of high- performance monodispersed chitosan microparticles for methyl orange adsorption Rsc Advances 5 78352-60

[31] Ma Z, Garrido-Maestu A and Jeong K C 2017 Application, mode of action, and in vivo activity of chitosan and its microand nanoparticles as antimicrobial agents: A review Carbohydrate Polymers 176 257-65

[32] Putri F A and Kennedy J F 1997 Application of chitin and chitosan Carbohydrate Polymers 34 414-

[33] Xu J, Xu X, Zhao H and Luo G 2013 Microfluidic preparation of chitosan microspheres with enhanced adsorption performance of copper(II) Sensors \& Actuators B Chemical 183 201-10

[34] Zhao H, Xu J, Lan W, Wang T and Luo G 2013 Microfluidic production of porous chitosan/silica hybrid microspheres and its $\mathrm{Cu}(\mathrm{II})$ adsorption performance Chemical Engineering Journal 229 82-9

[35] Zhang L, Han T, Xuan J, Xu H and Wang P 2018 A microfluidic module for preparing droplets on a large scale. 